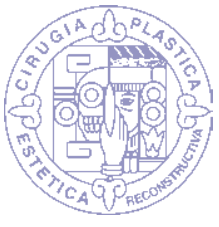

Caso clínico

doi: 10.35366/94379

https://dx.doi.org/10.35366/94379

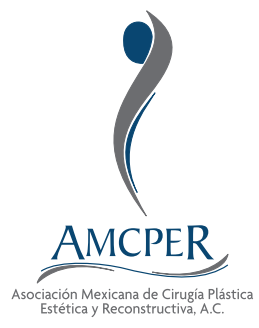

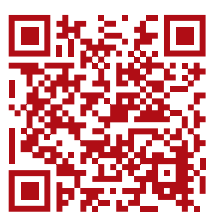

Palabras clave: Tumor glómico, tumor subungueal, diagnóstico, resección, tratamiento quirúrgico.

Keywords: Glomus tumor, subungual tumor, diagnosis, resection, surgical treatment.
${ }^{*}$ Médico adscrito de Cirugía Plástica y Reconstructiva.

* Médico Residente de Cirugía General.

Hospital Regional «General Ignacio Zaragoza», ISSSTE. Ciudad de México.

Los autores de este artículo no tienen conflicto de intereses que declarar.

\section{Recibido:}

18 noviembre 2018 Aceptado para publicar: 16 octubre 2019

\title{
Tumor glómico subungueal
}

\author{
Subungual glomus tumor
}

\section{Dra. Claudia Eugenia Milla-Bello, ${ }^{*}$ Dra. Maricarmen Danae Orozco-Bustos, ${ }^{\ddagger}$ Dr. Jesús Fernando Nagore-Ancona ${ }^{\ddagger}$}

\section{RESUMEN}

Los tumores glómicos son neoplasias benignas poco frecuentes. Constituyen de 1 a $5 \%$ de los tumores de la mano, con predominio en el sexo femenino 3:1. Presentamos el caso de una mujer de 46 años con una tumoración dolorosa subungueal del pulgar derecho de ocho años de evolución. Los médicos que la atendieron en forma inicial sospecharon de un melanoma, por lo que le realizaron una biopsia, con resultados negativos para malignidad. Posteriormente fue enviada al Servicio de Dermatología, al ser valorada diagnosticaron un tumor glómico, por lo que fue enviada al Servicio de Cirugía Plástica para su tratamiento. Se le realizó un ultrasonido y la imagen encontrada fue compatible con el diagnóstico. Se efectuó la resección de la tumoración y el estudio de patología confirmó el diagnóstico. La evolución a un año fue satisfactoria, sin recidiva del tumor y con un buen aspecto estético del dedo. La prueba imagenológica utilizada para la detección es la ultrasonografía; sin embargo, la resonancia magnética ha demostrado ser de mayor utilidad para precisar la localización y tipo de tumor. Un mal diagnóstico propicia tratamientos inadecuados, con riesgo para la salud, por lo cual se precisa una alta sospecha diagnóstica para ser identificado y tratado de manera adecuada.

\section{ABSTRACT}

Glomus tumors are rare benign neoplasms. They constitute $1-5 \%$ of tumors of the hand, predominantly in females 3:1. We present the case of a 46-year-old woman with a painful subungual right thumb tumor of eight years of evolution. The doctors who treated her initially, suspected a melanoma; so, she underwent a biopsy, with negative results for malignancy. Afterwards, she was sent to the dermatology department, when she was assessed they diagnosed a glomus tumor, so she was sent to the plastic surgery service for treatment. They performed an ultrasound; the image found was compatible with the diagnosis. The tumor was resected; the pathology study confirmed the diagnosis. The evolution at one year was satisfactory, without recurrence of the tumor and with a good aesthetic appearance of the finger. The imaging test used for detection is ultrasonography; however, magnetic resonance imaging has proven to be more useful to specify the location and type of tumor. A bad diagnosis leads to inadequate treatments, risking one's health. It requires a high diagnostic suspicion to be identified and treated properly.

\section{INTRODUCCIÓN}

L os tumores glómicos son neoplasias benignas de tipo mesenquimatoso poco frecuentes, que derivan de los cuerpos glómicos neuromioarteriales encargados de la regulación del flujo sanguíneo local y de la termorregulación. Constituyen de 1 a 5\% de los tumores de la mano, con predominio del sexo femenino con una relación 3:1 y se presentan con mayor frecuencia en la cuarta y quinta década de la vida. Se localizan en las extremidades superiores hasta en $80 \%$ de los casos y la región subungueal es la más afectada. ${ }^{1,2}$
Los tumores glómicos se clasifican en dos tipos: tumores solitarios o múltiples y el solitario es el más frecuente. La variante múltiple es más rara y no suele ser dolorosa y está asociada con patrones hereditarios de tipo autosómico dominante que aparece en edades más tempranas. $^{2,3}$

El glomus o cuerpo glómico es una estructura situada en la unión dermoepidérmica entre el extremo terminal de las arteriolas y el inicio de las vénulas unidas entre sí por vasos anastomóticos denominados canales de Sucquet-Hoyer, sin interposición de capilares intermedios. Tiene la función de controlar el calibre de los pequeños 
vasos para regular la circulación superficial y con ello la termorregulación, por lo que está muy inervado. De ahí la denominación de aparato neuromioarterial. , $^{3,4}$

El propósito de este trabajo es presentar el caso de una mujer con un tumor glómico subungueal con un mal diagnóstico inicial que retrasó el tratamiento definitivo. Mencionamos las bases para la identificación de este tipo de lesiones, así como del diagnóstico diferencial.

\section{CASO CLÍNICO}

Mujer de 46 años, de profesión médico, sin antecedentes personales patológicos relevantes para el padecimiento. En 2005 presentó una tumoración de color azul en la lúnula del pulgar derecho de $2 \mathrm{~mm}$ de diámetro, acompañada de dolor intenso punzante, de breve duración, sin predominio de horario. En 2014 fue valorada por el Servicio de Medicina Interna, por el antecedente de residencia en zona tropical (Chiapas) de ocho años, iniciaron tratamiento para onicomicosis durante tres meses con fluconazol, sin tener mejoría alguna. Al año siguiente, dada la persistencia de la sintomatología, fue enviada a oncología quirúrgica bajo la sospecha de melanoma. Se realizó toma de biopsia con resultado negativo a malignidad. Cursó con mejoría clínica tres meses después de la intervención quirúrgica y presentó nuevamente sintomatología de características similares, pero de mayor intensidad. Refería utilizar hielo local para remisión del dolor durante esos episodios, con mejoría parcial. En 2016 fue valorada por el Servicio de Dermatología, que sugirió se trataba de un tumor glómico y fue enviada al Servicio de Cirugía Plástica para manejo quirúrgico.
Al examen físico encontramos deformidad de la uña izquierda con elevación en el centro desde la matriz, sin cambios de coloración, dolorosa a la manipulación, sin alteración en los arcos de movimiento y con adecuado Ilenado capilar. Solicitamos ultrasonido que reportó lesión compatible con tumor glómico. Realizamos escisión y biopsia de la tumoración, conservando la matriz y el lecho ungueal. La evolución inmediata fue satisfactoria, sin complicaciones. El reporte del estudio de patología fue de tumor glómico de tipo mixoide.

En el seguimiento a un año del evento quirúrgico, la paciente expresó estar satisfecha con los resultados estéticos y estaba completamente asintomática.

\section{DISCUSIÓN}

El rasgo distintivo de estos tumores es que son habitualmente crónicos y localizados, mayormente, subungueales; además, pueden transcurrir varios años para que presenten síntomas incapacitantes. Su clínica se describe con alta sensibilidad de las pruebas y signos, pero con pobre especificidad (Tabla 1). El retraso en el diagnóstico se reporta con una media de siete años, pero con casos reportados de hasta 40 años. Al tratarse de entidades raras, los diagnósticos diferenciales son muy variados (Tabla 2). Con frecuencia se diagnostican erróneamente y esto favorece que se implementen tratamientos inadecuados que representan un riesgo para la salud. En una serie de cuatro casos, Gargollo Orvañanos y colaboradores, ${ }^{5}$ mencionan que las recurrencias se presentan en 10 a $20 \%$ de los casos y pueden ser secundarias a resecciones incompletas, falta de resección en la cirugía inicial o tumores de nueva aparición. La paciente

\section{Tabla 1: Clínica del tumor glómico.}

1. Signo de Love. Dolor agudo a la presión puntiforme sobre la tumoración y ausencia de dolor con la misma presión en áreas adyacentes al tumor

2. Prueba del frío. Dolor al aplicar agua fría o alcohol etílico

3. Signo de Hildreth. Desaparición del dolor al realizar isquemia en el brazo o en la base del dedo

4. Prueba de transiluminación. En un ambiente oscuro se proyecta luz a través del pulpejo para visualizar la zona roja, opaca característica de la lesión 
Tabla 2: Diagnósticos diferenciales del tumor glómico.

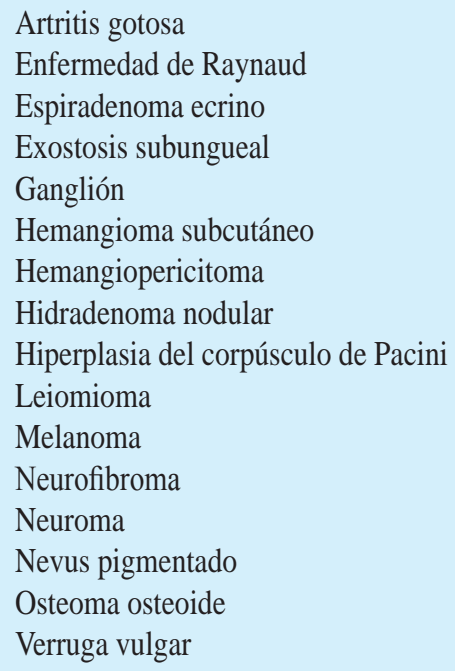

de este caso tenía ocho años de evolución y no la habían podido diagnosticar, además la biopsia que le realizaron no fue de utilidad para este propósito. El diagnóstico lo hizo un dermatólogo y fue confirmado con el estudio de patología de la biopsia que enviamos.

La resonancia magnética se describe como el estudio imagenológico de mayor relevancia diagnóstica; sin embargo, la ecografía preoperatoria puede ser de gran utilidad previo al tratamiento definitivo, que por excelencia es la escisión quirúrgica completa. ${ }^{6,7} \mathrm{No}$ sotros le indicamos un ultrasonido y con la imagen obtenida confirmamos el diagnóstico preoperatorio.

La tríada sintomática clásica consiste en dolor paroxístico, intolerancia al frío y localización explícita del dolor en la zona del tumor, que está presente sólo en un mínimo porcentaje de los casos. En la literatura se describe la prueba del frío, con una sensibilidad y especificidad del 100\%; no obstante, de manera paradójica, en este caso, la paciente refería remisión del dolor con la aplicación de compresas de hielo y esto se puede explicar por la vasoconstricción y reducción de migración de moduladores del dolor. ${ }^{8,9}$

Los tumores glómicos malignos son raros, la mayoría de ellos son agresivos sólo de ma- nera local y con recurrencias locales múltiples. Se ha observado que se originan a partir de un tumor glómico benigno, aunque aún no está claro el mecanismo por el cual ocurre esta transformación. Cerca de 1\% se consideran malignos, cuyas características histopatológicas incluyen: tamaño mayor a $2 \mathrm{~cm}$, localización en planos profundos, alto grado de mitosis y prevalencia de figuras mitóticas atípicas. Si se establecen los criterios, el riesgo de metástasis es mayor a $25 \%$, por lo que se recomienda el estudio anatomopatológico de manera rutinaria. $^{10-12}$

\section{CONCLUSIÓN}

El tumor glómico es una entidad patológica que requiere de una alta sospecha diagnóstica para ser identificado y tratado de manera adecuada. Dentro de las pruebas imagenológicas, el ultrasonido es una opción accesible y económica, útil antes del manejo definitivo, que es la escisión quirúrgica completa. Asimismo, el estudio histopatológico es obligatorio.

\section{REFERENCIAS}

1. Henao E, Gómez O. Tumor glómico subungueal: reporte de un caso. Rev Med Sanitas 2013; 16 (2): 38-41.

2. De Carli P, Lupotti C, Illarramendi A. Tumor glómico en dedos de la mano. Rev Asoc Arg Ortop y Traumatol 1996; 61 (1): 34-40.

3. Ramos GA, Herbas RO. Tumor glómico. Presentación de un caso con topografía poco usual. Rev Cent Dermatol Pascua 2006; 15 (3): 187-189.

4. Celester Barreiro G, Prego Bestilleiro A, González del Castillo F, Tobío Martel RM, Vázquez García J, Arriaza Loureda R. Tumores glómicos en el miembro superior. Revisión de la literatura e informe de 14 casos. Rev Iberoam Cir Mano 2016; 44 (2): 69-77.

5. González G, Case M, Orvañanos G, Orvañanos G, Gómez G, Galindo R et al. Experiencia en tumores glómicos en el Hospital General "Dr. Manuel Gea González" (México). Reporte de casos. Cir Plast Iberolatinoam 2010; 36 (4): 369-374.

6. Trehan SK, Athanasian EA, Dicarlo EF, Mintz DN, Daluiski A. Characteristics of glomus tumors in the hand not diagnosed on magnetic resonance imaging. J Hand Surg Am 2015; 40 (3): 542-545.

7. Theumann NH, Goettmann S, Le Viet D, Resnick D, Chung CB, Bittoun J et al. Recurrent glomus tumors of fingertips: mr imaging evaluation. Radiology 2002; 223 (1): 143-151.

8. Chang P, Orellana E, Rosales D, Calderón G. Tumor glómico. Dermatol Rev Mex 2013; 57: 278-282. 
9. Samaniego E, Crespo A, Sanz A. Claves del diagnóstico y tratamiento del tumor glómico subungueal. Actas Dermosifiliogr 2009; 100: 875-882.

10. Rodríguez M, Calderón C, Ramos-Garibay A. Tumor glómico solitario. Presentación de un caso. Rev Cent Dermatol Pascua 2001; 10 (2): 77-79.

11. Torres González S, Ramos Garibay A, Lizárraga García C. Tumor glómico atípico. Revisión de la literatura a propósito de un caso. Dermatol Rev Mex 2006; 50: 109-114.

12. Polo C, Borda D, Poggio D, Asunción J, Peidro L. Glomus tumor of the hallux. Review of the literature and report of two cases. Foot Ankle Surg 2012; 18 (2): 89-93.

Correspondencia:

Dr. Jesús Fernando Nagore-Ancona

Hospital Regional «General Ignacio Zaragoza».

Calzada Gral. Ignacio Zaragoza Núm. 1711,

Col. Ejército Constitucionalista, 09220,

Iztapalapa, Ciudad de México, México.

E-mail: fer_nagore@hotmail.com 